\title{
Simulation of wedge water entry using smoothed particle hydrodynamics method
}

\author{
Mohammad Farsi, Parviz Ghadimi*, Rahim Zamanian, Abbas Dashtimanesh \\ Department of Marine Technology, Amirkabir University of Technology, Tehran, Iran \\ *Corresponding author E-mail: pghadimi@aut.ac.ir
}

\begin{abstract}
This article presents a study based on the smoothed particles hydrodynamics method, aiming at a numerical simulation of solid-fluid coupling in a free surface flow. Smoothed Particle Hydrodynamics method (SPH) represents a very interesting alternative method to classical mesh-based methods such as Finite-Volume, Finite-Difference or FiniteElement Methods. The SPH scheme is first described and discussed through its formulations. Two test cases of wedge water entry are presented. To accomplish this, 2D-SPH models in conjunction with turbulence model are implemented and MLS density filter is utilized to remove pressure fluctuations. Pressure distribution, time history of pressure and free surface level are provided. In order to validate the obtained free surface, free surface of dam break problem is simulated and compared with experimental result. Also, the obtained pressure distribution is compared with the analytical result of similarity solution.
\end{abstract}

Keywords: Water Entry; Wedge; SPH Method; Pressure Distribution; Free Surface Level

\section{Introduction}

Water impact pressure arising during the impact particularly during the initial stages, is crucial in the design of marine structures. The earliest study published on the determination of water entry impact forces dates back to 1929 with Von Karman [1]. This study used simple principles such as conservation of momentum and the concept of added mass. In 1932, the theoretical solution of water entry problem was continued with the work of Wagner [2] who analyzed the vertical water impact of a two-dimensional wedge with small deadrise angle. In 1934, Sedov [3] expanded Wagner's solution to the impact of two-dimensional wedge with large deadrise angle.

There are many numerical methods for simulation of the water entry problem [4, 5, 6 and 7]. Common methods of modeling are grid-based. However, these methods encounter computational difficulties when phenomena such as flow separation, surface piercing or large movement are involved in the simulation. Then, it becomes difficult to capture the movement and fluid response to a moving object. The computational method presented in this paper is SPH which is a mesh free method. This technique was initially developed for the study of particle motion in highly turbulent phenomena within astrophysics by Gringold and Monaghan [8] and Lucy [9]. After that, SPH method was adopted for free surface flows, flow through porous media, impact and fracture problem, water waves generation, and fluidstructure interaction [10 to17].

There are some studies on water entry problem by SPH. Oger et al. [18] simulated wedge water entry by SPH and introduced a new technique to evaluate the pressure on solid boundaries. That study was involved a spatially varying resolution based on variable smoothing length technique, and a new formulation of the equations was proposed. Oger et al. [19] also simulated water entry of a sphere at high impact velocity. They showed that SPH simulations can be performed on supercomputers. They used hundreds of millions of particles to simulate the sphere water entry. Gong et al. [20] also studied the hydrodynamics of a two dimensional wedge water entry based on SPH technique. They proposed a non-reflecting boundary condition to reduce the reflection of sound waves. A series of tests with different initial velocities was performed and maximum force for different conditions was obtained. Vepa et al. [21] predicted pressure distribution of a 2D rigid cylinder during water entry by SPH method. They presented the time history of pressure distribution and maximum pressure. They showed that by increasing particles, the accuracy of solutions is increased. Veen and Gourlay [22] developed a two dimensional SPH algorithm and applied to the problem of ship slamming. Vandame et al. [23] simulated the entry and exit of a floating object using WCSPH technique. They examined the water impact, hydrodynamic forces, fluid motions and movement of objects. They simulated a $2 \mathrm{D}$ wedge 
drop test and the achieved results showed good agreement with experimental data. Moreover, they presented velocity field and free surface profile.

In this article, water impact of 2D wedge is simulated. For this purpose, a 2D-SPH model in conjunction with a turbulence model is implemented. Furthermore, MLS density filter is utilized to remove pressure fluctuations. Time history of pressure, pressure distribution and free surface elevation are also presented.

\section{Numerical procedure}

\subsection{SPH formulation}

Smoothed Particle Hydrodynamics was originally developed as a probabilistic mesh free particle method for simulating astrophysical problems [8, 9]. It was later modified and applied to continuum solid and fluid mechanics [24]. The SPH method is based on interpolation theory. By means of an interpolation function, points can be used to discretize partial differential equations without any underlying mesh.

The concept of integral representation of a generic function $\boldsymbol{f}(\overrightarrow{\boldsymbol{x}})$ of the position vector $\overrightarrow{\boldsymbol{x}}$ that is used in the SPH method originates from the following identity [25]

$f(\vec{x})=\int_{\Omega} f\left(\vec{x}^{\prime}\right) \delta\left(\vec{x}-\vec{x}^{\prime}\right) d \vec{x}^{\prime}$

where function $\boldsymbol{f}(\overrightarrow{\boldsymbol{x}})$ is defined on the n-dimensional domain $\Omega, \overrightarrow{\boldsymbol{x}}^{\prime}$ is the elementary volume surrounding the point $\overrightarrow{\boldsymbol{x}}^{\prime}$ located in the neighborhood of point $\overrightarrow{\boldsymbol{x}}$, and $\boldsymbol{\delta}\left(\overrightarrow{\boldsymbol{x}}-\overrightarrow{\boldsymbol{x}}^{\prime}\right)$ is the Dirac delta function defined as

$\delta\left(\vec{x}-\vec{x}^{\prime}\right)= \begin{cases}1 & \vec{x}=\vec{x}^{\prime} \\ 0 & \vec{x} \neq \vec{x}^{\prime}\end{cases}$

If the Delta function kernel $\boldsymbol{\delta}\left(\overrightarrow{\boldsymbol{x}}-\overrightarrow{\boldsymbol{x}}^{\prime}\right)$ is replaced by a smoothing function $\boldsymbol{W}\left(\overrightarrow{\boldsymbol{x}}-\overrightarrow{\boldsymbol{x}}^{\prime}, \boldsymbol{h}\right)$, the integral representation of $\boldsymbol{f}(\overrightarrow{\boldsymbol{x}})$ is then given by [25]

$f(\vec{x})=\int_{\Omega} f\left(\vec{x}^{\prime}\right) W\left(\vec{x}-\vec{x}^{\prime}, h\right) d \vec{x}^{\prime}$

where $\mathrm{W}$ is the so-called smoothing kernel function. In this formula, $h$ represents the smoothing length of the smoothing function W. In this work, cubic spline is used as a kernel function which is defined as [25]

$\boldsymbol{W}(\boldsymbol{r}, \boldsymbol{h})=\boldsymbol{\alpha}_{\boldsymbol{D}}\left\{\begin{array}{lr}1-\frac{3}{2} \boldsymbol{q}^{2}+\frac{3}{4} \boldsymbol{q}^{3} & 0 \leq \boldsymbol{q} \leq 1 \\ \frac{1}{4}(2-\boldsymbol{q})^{3} & 1 \leq \boldsymbol{q} \leq 2 \\ 0 & \boldsymbol{q} \geq 2\end{array}\right.$

where $\boldsymbol{\alpha}_{\boldsymbol{D}}$ is $\frac{10}{7 \pi \boldsymbol{h}^{2}}$ in two dimensional problems, $\boldsymbol{q}=\boldsymbol{r} / \boldsymbol{h}$ with $\boldsymbol{r}$ is the distance between particles $i$ and $j$.

Equation (3) is a continuous function and can be approximated by a summation over the mass elements. This procedure gives the summation interpolation (Fig.1) as in

$\boldsymbol{f}\left(\overrightarrow{x_{l}}\right)=\sum_{j=1}^{N} m_{j} \frac{f_{j}}{\rho_{j}} W_{i j}$

Where $m_{j}$ and $\rho_{j}$ denote the mass and density of particle $j$, respectively. $N$ is the number of neighboring particles for basic particle $i$. 


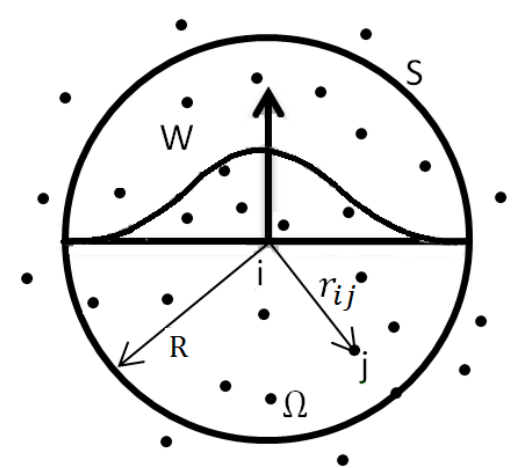

Fig.1: Particle approximation using particles within the support domain of the smoothing function W for particle i [25]. The support domain is circular with a radius of $\mathrm{R}$.

\subsection{Governing equations}

The rate of change in density or the continuity equation, is written as

$\frac{D \rho}{D t}=-\rho \nabla \cdot v$

Continuity equation for particle $i$ can be presented in the SPH formulation as

$\frac{D \rho_{i}}{D t}=-\sum_{j=1}^{N} \boldsymbol{m}_{j}\left(u_{j}-u_{i}\right) \cdot \nabla W_{i j}$

where the sum extends over all neighboring particles and $\mathrm{W}$ is the smoothing kernel evaluated at the distance between particles $i$ and $j$.

The momentum conservation equation in the continuum field is given as

$\frac{D v}{D t}=-\frac{1}{\rho} \nabla p+g+\Theta$

where $v, p$ and $\rho$ are the velocity, pressure and density, respectively. $\boldsymbol{g}=(0,0,9.81) \boldsymbol{m s}^{-2}$ is the gravitational acceleration and $\Theta$ refers to the diffusion terms. Based on Laminar viscosity and Sub-Particle Scale (SPS) Turbulence, the SPH formulation of the momentum equations can also be derived by a particle approximation procedure as in

$\frac{d \overrightarrow{v_{l}}}{d t}=-\sum_{j} m_{j}\left(\frac{p_{j}}{p_{j}^{2}}+\frac{p_{i}}{p_{i}^{2}}\right) \nabla_{i} W_{i j}+\vec{g}+\sum_{j} m_{j}\left(\frac{4 v_{0} \vec{r}_{i j} \nabla_{i} W_{i j}}{\left(\rho_{i}+\rho_{j}\right)\left|\overrightarrow{r_{l}}\right|^{2}}\right) \vec{v}_{a b}+\sum_{j} m_{j}\left(\frac{\tau_{j}}{p_{j}^{2}}+\frac{\tau_{i}}{p_{i}^{2}}\right) \nabla_{i} W_{i j}$

The eddy viscosity assumption is often used to model the SPS stress tensor using Favre-averaging of $\frac{\tau_{i j}}{\rho}=$ $v_{t}\left(2 S_{i j}-\frac{2}{3} k \delta_{i j}\right)-\frac{2}{3} C_{I} \Delta^{2} \delta_{i j}\left|S_{i j}\right|^{2}$, where $\tau_{i j}$ is the sub-particle stress tensor, $v_{t}=\left[C_{s} \Delta l\right]^{2}|S|$ is the turbulence eddy viscosity, $k$ is the SPS turbulence kinetic energy, $C_{s}=0.12$ is the Smagorinsky constant, $C_{I}=0.0066, \Delta l$ is the particle-particle spacing and $|S|=\left(2 S_{i j} S_{i j}\right)^{0.5}$, where $S_{i j}$ is the element of SPS strain tensor [26, 27 and 28].

\subsection{Time stepping scheme}

The SPH equations are integrated with a slight modification of the standard predictor-corrector method which is second order accuracy in time [29]. The predictor step is given by

$v^{\frac{1}{2}}=v^{0}+\frac{\Delta t}{2} f^{0}, \quad r^{\frac{1}{2}}=r^{0}+\frac{\Delta t}{2} v^{\frac{1}{2}}, \quad e^{1 / 2}=e^{0}+\frac{\Delta t}{2} \dot{e}^{2}$

where in practice $f^{0} \approx f^{-\frac{1}{2}}$ was used and $\dot{e}^{0} \approx \dot{e}^{-\frac{1}{2}}$ to give a one-step method. The rates of change of these quantities are then computed via the SPH summations using the predicted values at the half step, i.e.

$f^{1 / 2}=f\left(r^{\frac{1}{2}}, v^{\frac{1}{2}}\right), \quad \dot{e}^{1 / 2}=\dot{e}\left(r^{\frac{1}{2}}, v^{\frac{1}{2}}\right)$

The corrector step is given by

$v^{*}=v^{0}+\frac{\Delta t}{2} f^{1 / 2}, \quad r^{*}=r^{0}+\frac{\Delta t}{2} v^{*}, \quad e^{*}=e^{0}+\frac{\Delta t}{2} \dot{e}^{1 / 2}$

and finally 
$v^{1}=2 v^{*}-v^{0}, \quad r^{1}=2 r^{*}-r^{0}, \quad e^{1}-2 e^{*}-e^{0}$

In this scheme, the position updates in both the predictor and corrector steps use the updated value of velocity. This effectively means that the position is updated using both the first and second derivatives.

\subsection{Moving the particles}

In numerical modeling, filtering techniques are utilized for reduction of the numerical error in order to enhance the stability and convergence. The XSPH correction is utilized to move particles [30]

$\frac{d \overrightarrow{r_{i}}}{d t}=\vec{v}_{i}+\varepsilon \sum_{j} \frac{m_{j}}{\bar{\rho}_{i j}} \vec{v}_{i j} W_{i j}$

where $\varepsilon=0.5$ and $\bar{\rho}_{i j}=\left(\rho_{i}+\rho_{j}\right) / 2$. Particle $i$ is moved using the XSPH variant with a velocity equal to the average velocity in its neighborhood. This method is also instrumental in keeping the particles equally spaced.

\subsection{Boundary conditions}

Interaction between fluid particles and boundary particles must be controlled, accurately. Dynamic boundary condition is used in this study which was developed by Dalrymple and Knio [31]. With this boundary condition, boundary particles must satisfy the continuity and momentum equations, same as the fluid particles. They remain fixed in position (fixed boundaries) or move according to some externally imposed function (wedge).

\subsection{Validation of code}

In order to validate the code, the dam break problem is considered. The geometry of the problem is shown in Fig.2. Free surface elevation was measured at point $\mathrm{H}$ and compared against the experimental data of Kleefsman et al. [32]. The result of this simulation is presented in Fig.3, which displays good agreement between the numerical and experimental results. Furthermore, two different simulations comprising of 52356 particles and 94045 particles are conducted which are presented in Fig.3 and identified as Numeric 1 and Numeric 2, respectively. It is observed that by increasing the number of particles, the accuracy of simulations have improved, relatively.

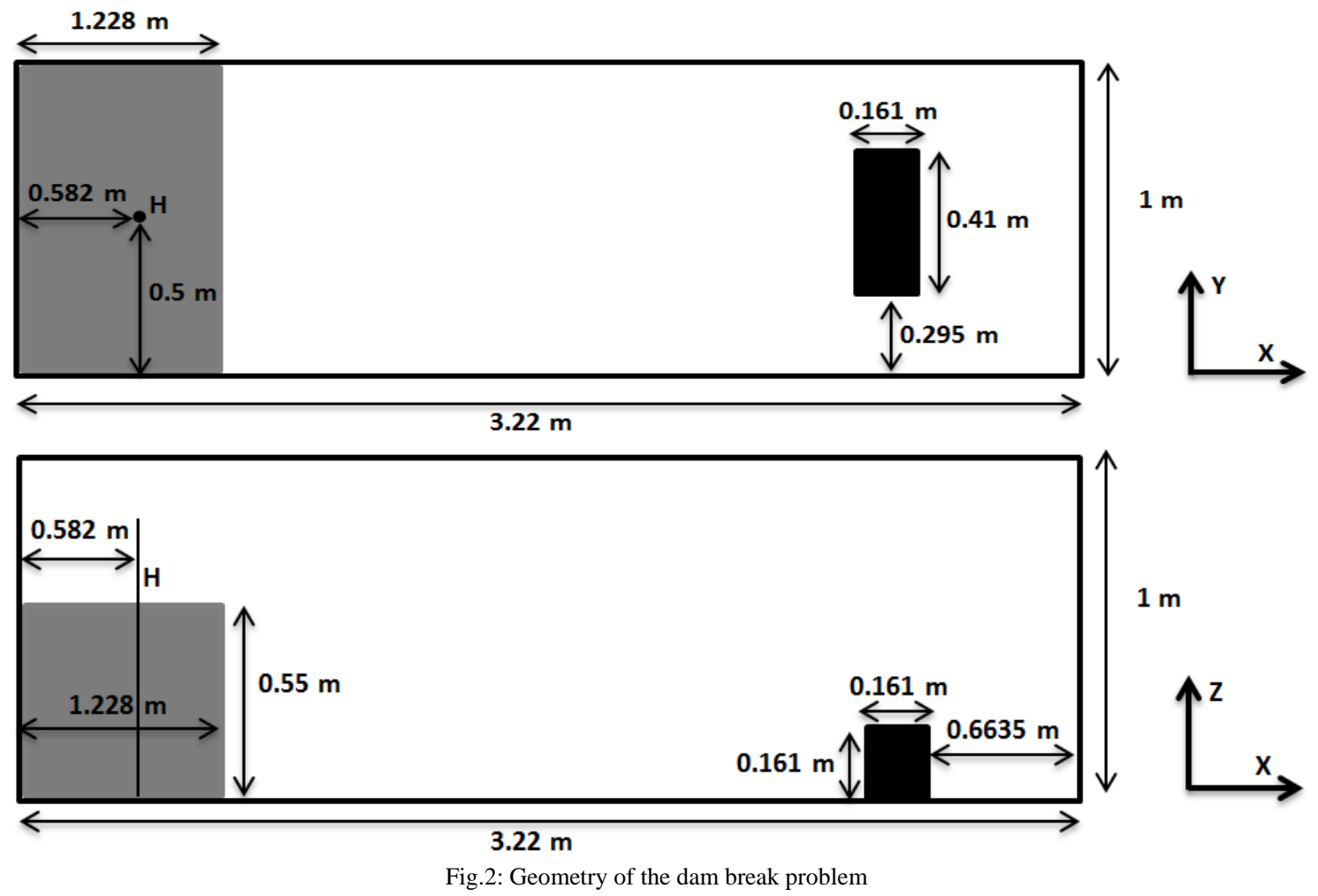




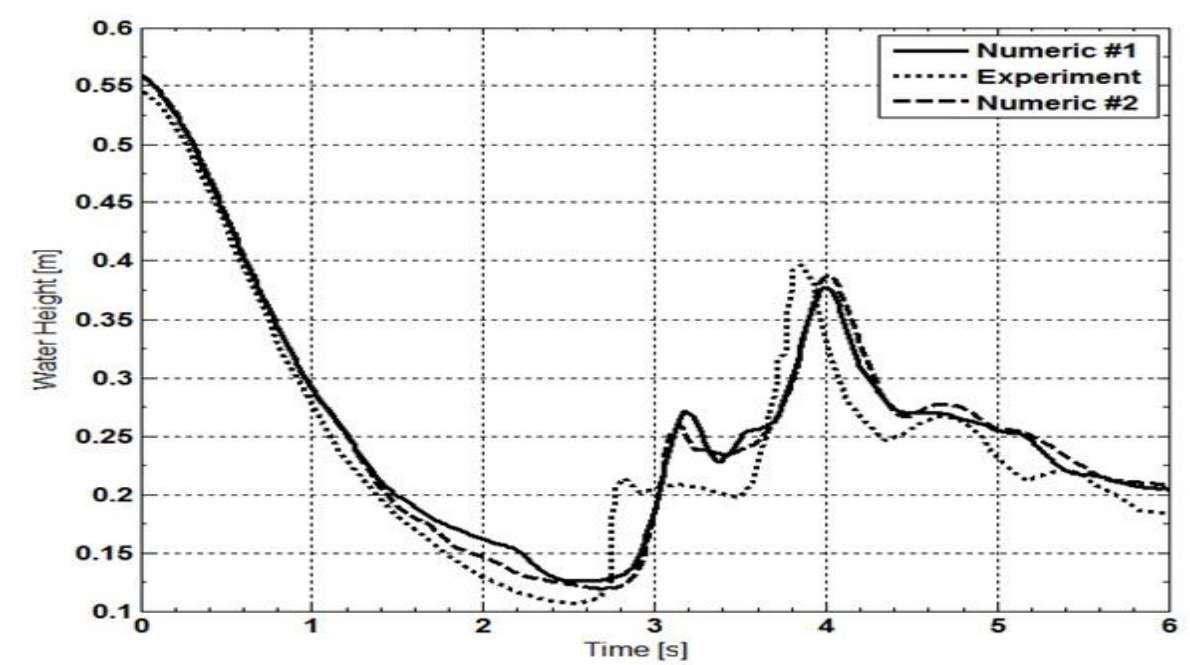

Fig.3: Numerical results versus Experimental data by Kleefsman et al. [32] and computational cases of Numeric \#1: 52356 particles and Numeric \#2: 94045 particles.

\section{Results}

In current work, pressure distribution, time history of pressure and free surface elevation are studied. Two tests including constant entering velocity of two wedges are investigated which are listed in table 1. Figure 4 shows initial configuration of a typical simulation. The width of the wedge is $0.2 \mathrm{~m}$. Both width and height of the computational domain are equal to $1 \mathrm{~m}$. The height of water level in the numerical tank is $0.5 \mathrm{~m}$. The distance between apex and free surface level at initial time is $0.01 \mathrm{~m}$. In order to complete simulation of water entry, both gravity and viscosity are included in formulations. Due to computational limitations, the number of particles considered in these studies is about 200,000 particles.

Table 1: Description of tests

\begin{tabular}{|c|c|c|}
\hline Test & Velocity & Deadrise angle \\
\hline 1 & Constant $=2 \mathrm{~m} / \mathrm{s}$ & 30 \\
\hline 2 & Constant $=2 \mathrm{~m} / \mathrm{s}$ & 45 \\
\hline
\end{tabular}

The distance of first and second probe for pressure is located at $2 \mathrm{~cm}$ and $9 \mathrm{~cm}$ from apex, respectively. Pressure distribution is presented in Fig.5. There is favorable agreement between similarity solution [33] and SPH method. Figure 6 presents free surface at $\mathrm{t}=0.0167 \mathrm{~s}$. At this time, the second probe captures the impact pressure and fluid particles touches the probe location. Moreover, the location of second probe is also shown in Fig. 6 with blue dashed line.

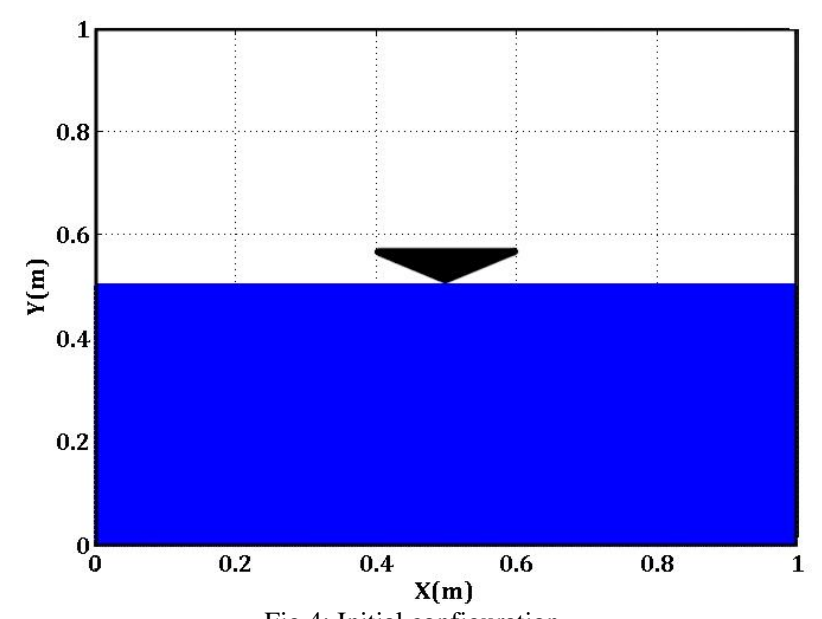

Fig.4: Initial configuration. 


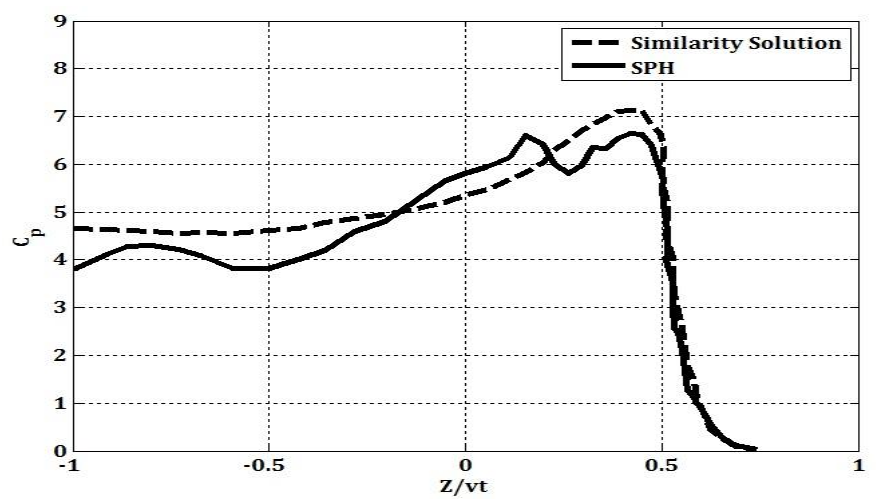

Fig.5: Pressure Distribution for case 1

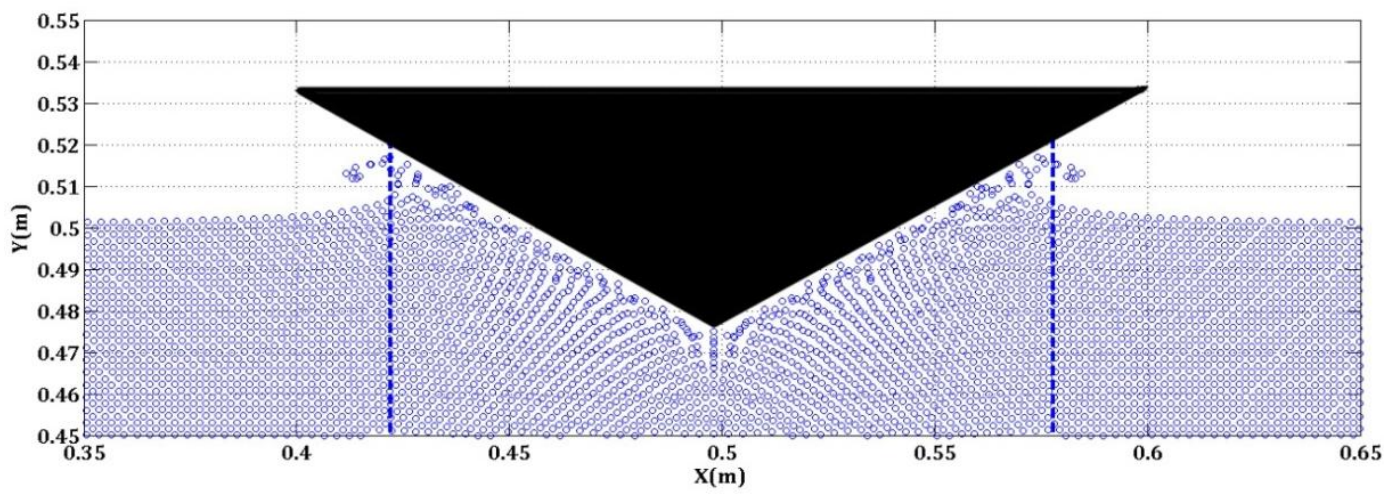

Fig.6: Free surface at $\mathrm{t}=0.0167 \mathrm{~s}$. The blue dashed line illustrates the location of second probe, $\beta=30^{\circ}$.

Case 2 is considered water entry of wedge at 45 degrees deadrise angle. All initial configurations are the same as case 1. Pressure distribution of this case is presented in Fig.8. The pressure distribution for this wedge by similarity solution [33] is also shown in Fig.8. There is favorable agreement between similarity solution and SPH method. Figure 9 presents free surface at $\mathrm{t}=0.0262 \mathrm{~s}$. At this time, fluid particles touch the probe location. Moreover, the location of second probe is also shown in Fig.9 with blue dashed line.

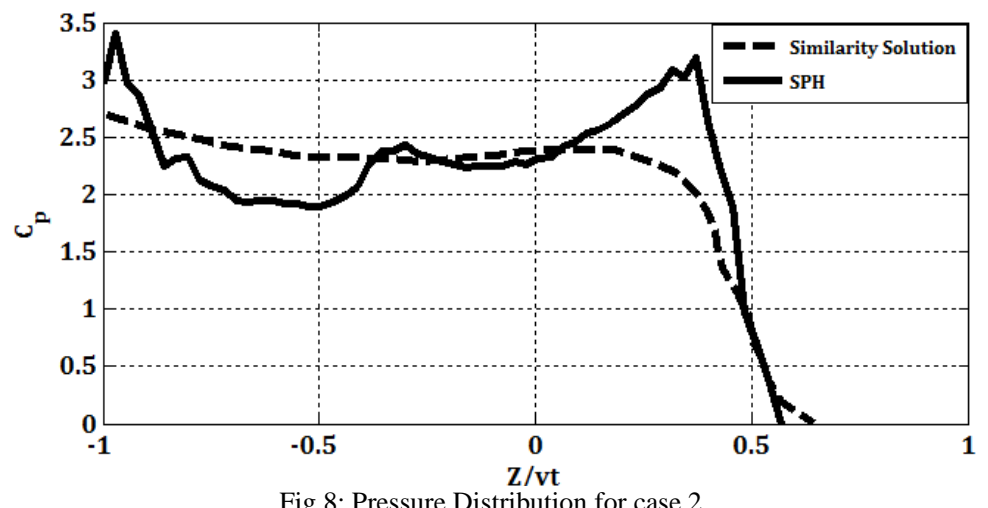

Fig.8: Pressure Distribution for case 2 


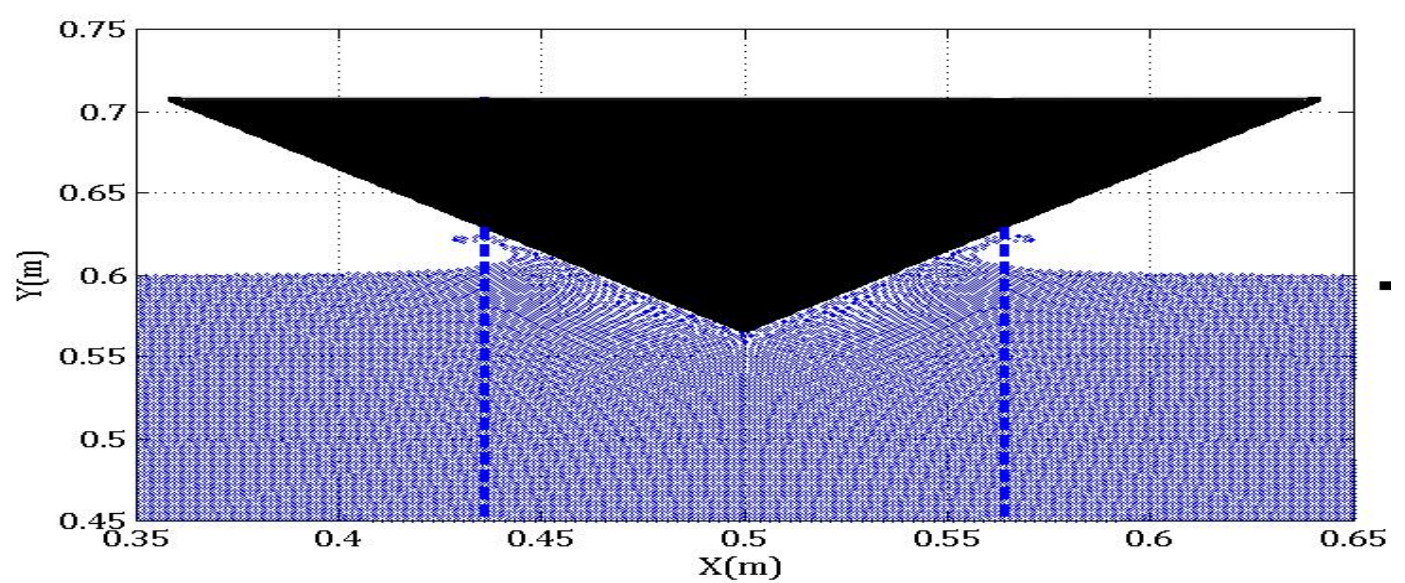

Fig.9: Free surface at $\mathrm{t}=0.0262 \mathrm{~s}$. The blue dashed line illustrates the location of second probe, $\beta=45^{\circ}$

\section{Conclusions}

In this paper, water entry of a wedge is simulated by the SPH method. Two test cases were considered. The first case is a wedge of 30 degrees deadrise angle and the second is a wedge of 45 degrees deadrise angle. For this purpose, 2DSPH models in conjunction with turbulence model are implemented and MLS density filter is utilized to remove pressure fluctuations. Time history of pressure, pressure distribution and free surface levels are presented. For validation of the obtained free surface, free surface of dam break problem is modeled and compared with experimental data. Also, the obtained pressure distribution is compared against the analytical solution (similarity solution). Favorable agreements have been achieved in both validation cases.

\section{References}

[1] Von Karman T. The impact of seaplane floats during landing. NACA TN 321, National Advisory Committee for Aeronautics, Washington, USA, October 1929

[2] Wagner H. Trans. phenomena associated with impacts and sliding on liquid surfaces. Math Mech1932;12(4):193-215.

[3] Sedov L. The impact of a solid body floating on the surface of an incompressible fluid Report 187, CAHI, Moscow, 1934.

[4] Greenhow, M., and Moyo, S. (1997). "Water entry and exit of horizontal circular cylinders.”Philos. Trans. R. Soc. A, 355(1724), 551-563.

[5] Yan, S., and Ma, Q. W. (2007). "Numerical simulation of fully nonlinear interaction between steep waves and 2D floating bodies using the QALE-FEM method.”J. Comput. Phys., 221(2), 666-692.

[6] Zhang, Y. L., et al. (2010). “A level set immersed boundary method forwater entry and exit.” Commun.Comput. Phys., 8(2), $265-288$.

[7] Zhao, R., Faltinsen, O., and Aarsnes, J. (1997). "Water entry of arbitrarytwo-dimensional sections with and without flow separation." 21 st Symp.on Naval Hydrodynamics, National Academies Press, Washington, DC.

[8] Gringold, R., and Monaghan, J. J. (1977). "Smoothed particle hydrodynamics: Theory and application to non-spherical stars."Mon. Not. R.Astron. Soc., 181, 375-388.

[9] Lucy, L. (1977). “A numerical approach to testing of the fusion process.”Astron. J., 88, 1013-1024.

[10] Dalrymple, R. A. (2007). "Using Smoothed Particle Hydrodynamics for Waves." 4th Int. Asian and Pacific Coasts Conf., World Scientific Publishing, Singapore.

[11] Dalrymple, R. A., et al. (2009). "Smoothed particle hydrodynamics for water waves."Advances in numerical simulation of nonlinear water waves, Q. Ma, ed., World Scientific Publishing, Singapore.

[12] Ferrari, A. (2010). "SPH simulation of a free surface flow over a sharp crested weir.”Adv. Water Resour., 33(3), $270-276$.

[13] Gómez-Gesteira, M., Cerqueiro, D., Crespo, C., and Dalrymple, R. A. (2005). "Green water overtopping analyzed with a SPH model." Ocean Eng., 32(2), 223-238.

[14] Gómez-Gesteira, M., and Dalrymple, R. A. (2004). "Using a 3D SPH method for wave impact on a tall structure.”J. Waterw. Port Coastal Ocean Eng., 130(2), 63-69.

[15] Gómez-Gesteira, M., Rogers, B. D., Dalrymple, R. A., and Crespo, A. J. C. (2010). "State-of-the-art of classical SPH for free surface flows." J. Hydraul. Res., 48, 6-27

[16] Monaghan, J. J. (1994). "Simulating free surface flows with SPH.” J. Comput. Phys., 110, 399-406.

[17] Rogers, B. D., Dalrymple, R. A., and Stansby, P. K. (2008). SPH modeling of floating bodies in the surf zone, ICCE, Hamburg, Germany.

[18] G. Oger *, M. Doring, B. Alessandrini, P. Ferrant, "Two-dimensional SPH simulations of wedge water entries", Journal of Computational Physics 213 (2006) 803-822.

[19] Guillaume Oger, David Le Touzé, Bertrand Alessandrini and Pierre Maruzewski,"A new parallelized 3D SPH model: resolution of water entry problems and scalability study".

[20] GONG Kai, LIU Hua, WANG Ben-long, "WATER ENTRY OF A WEDGE BASED ON SPH MODEL WITH AN IMPROVED BOUNDARY TREATMENT”, Journal of Hydrodynamics, 2009,21(6):750-757, DOI: 10.1016/S1001-6058(08)60209-7.

[21] K.S. Vepa, D. Van Nuffel, W. Van Paepegem, "PRESSURE PREDICTIONS DURING WATER ENTRY OF A 2D RIGID CYLINDER USINGSPH METHOD”. 
[22] Daniel Veen, Tim Gourlay, "A combined strip theory and Smoothed Particle Hydrodynamics approach for estimating slamming loads on a ship in head seas", Ocean Engineering 43 (2012) 64-71.

[23] Johan Vandamme; Qingping Zou; and Dominic E. Reeve, "Modeling Floating Object Entry and Exit Using Smoothed Particle Hydrodynamics".

[24] Monaghan, J. J. Smoothed particle hydrodynamics. Ann. Rev. Astronomy and Astrophysics. 1992, vol. 30, pp. 543-574.

[25] Liu, G. R. and Liu, M. B. Smoothed Particle Hydrodynamics - a Meshfree Particle Methods. World Scientific Publ, 2007.

[26] Issa, R., Numerical assessment of the Smoothed Particle Hydrodynamics gridless method for incompressible flows and its extension to turbulent flows, Ph.D. Thesis, University of Manchester Institute of Science and Technology (UMIST), 2004.

[27] Lo, E.Y.M. and Shao, S., Simulation of near-shore solitary wave mechanics by an incompressible SPH method, Applied Ocean Research, 24, 275-286, 2002.

[28] Pope, S.B., Turbulent Flows, Cambridge University Press, 2000.

[29] Monaghan, J.J., On the problem of penetration in particle methods. Journal of Computational Physics, 1989, 82 (1), 1- 15.

[30] Monaghan, J. J. On the problem of penetration in particle methods. Journal Comp Phys., 1989, vol. 82, pp. 1-15.

[31] Dalrymple, R. A. and Knio, O. SPH modelling of water waves. Proc. Coastal Dynamics, Lund, 2000.

[32] Kleefsman, K. M. T., Fekken, G., Veldman, A. E. P., Iwanowski, B. and Buchner, B. A volume of fluid based simulation method for wave impact problems. J. Comp. Phys., 2005, vol. 206, pp. 363-393.

[33] R. Zhao, O. Faltinsen, J. Aarsnes, "Water Entry of Arbitrary Two-Dimensional Sections with and Without Flow Separation", Twenty-First Symposium on Naval Hydrodynamics (1997). 